\title{
Aportes de los centros de investigación científica en ciencias biológicas al desarrollo sostenible de comunidades rurales mediante el turismo científico
}

Contributions of Biological Research Centers to Sustainable Development of Rural Communities Through Scientific Tourism

Alberth Humberto Rojas-Carranza ${ }^{1}$

Rojas-Carranza, A. H. Aportes de los centros de investigación científica en ciencias biológicas al desarrollo sostenible de comunidades rurales mediante el turismo científico. Tecnología en Marcha. Vol. 31 - Número Especial Movilidad Estudiantil 5. Octubre 2018. Pág 105-113.

DOI: https://doi.org/10.18845/tm.v31.i5.4088 


\title{
Palabras clave
}

Turismo; Turismo científico; Centros de investigación; comunidades rurales; Centro Soltis; Instituto Uiraçu.

\section{Resumen}

Los centros de investigación en ciencias biológicas tienen una función muy importante en el desarrollo científico y un impacto directo en la comunidad donde se asientan. Es claro, que en muchos casos ese impacto pasa desapercibido y no se valora la relación que existe entre ambos agentes, centro y comunidad, y las posibilidades que en materia de turismo científico pueden existir. En este artículo se describe la realidad de dos centros de investigación, uno en Brasil y el otro en Costa Rica. Tanto los centros como las comunidades en donde se encuentran, muestran características relevantes de tomar en cuenta al momento de relacionarlos con una actividad como el turismo científico. Aquí se describe en detalle y se pretende iniciar un proceso que llegue a una propuesta con fundamentación teórica más adelante, en el momento de desarrollar una práctica profesional.

\section{Keywords}

Tourism; Scientific Tourism; Research Centers; Rural communities; Soltis Center; Uiraçu Institute.

\begin{abstract}
Research centers have an important role in the scientific area and a direct impact on the community they are located. It is clear that in many cases this impact and the relationship between them appears unnoticed and is not valued. There are many ways to integrate both: centers and community in scientific tourism. This article describes the reality of two research centers, one in Brazil and one in Costa Rica. Both centers and communities where they are, show important features to take into account when relating them to an activity such as scientific tourism. These features are described in detail and seek to launch a process that comes to a theoretically supported proposal later.
\end{abstract}

\section{Introducción}

Este artículo es el resultado de una pasantía realizada en el marco del programa de Movilidad Estudiantil, llevado a cabo por el Instituto Tecnológico de Costa Rica a partir del año 2013 para favorecer la internacionalización de la educación de sus estudiantes.

El objetivo general de la pasantía fue describir mediante el análisis de un caso particular en Brasil y otro en Costa Rica, los aportes que mediante sus operaciones, los centros de investigación científica en ciencias biológicas brindan o podrían brindar a las comunidades rurales como factores de desarrollo sostenible, especialmente a través del turismo científico.

El turismo, como toda actividad productiva muy heterogénea y altamente dinámica en crecimiento ha presentado cambios importantes en el ámbito mundial que han favorecido la creación de nuevas tendencias o tipos de turismo, entre las cuales se pueden citar el geo turismo, el turismo de aventura, el turismo cultural, el turismo de negocios, el turismo de salud, el ecoturismo y el turismo científico, siendo este ultimo uno de los tipos de turismo menos conocidos y analizados académicamente. 
Según Bourlon y Mao [3] el turismo científico se define como el tipo de turismo que involucra directamente a investigadores que viajan por razones de trabajo o de experimentación por colaboraciones, intercambios internacionales, congresos, seminarios o coloquios. Al mismo tiempo, los autores mencionados indican que el relacionar el campo turístico con la actividad científica de un investigador puede presentar varios puntos de debate.

Bourlon, Mao y Osorio [2] muestran que el turismo de investigación científica tuvo sus inicios al principio de la década del 2000, y se considera una disciplina relativamente joven dentro del sector turístico. De acuerdo a estos mismos autores, esta actividad es también muy especializada.

A pesar de no ser creados específicamente para la recepción de turismo, al tomar como referencia la definición clásica de turismo brindada por la Organización Mundial de Turismo (OMT), la naturaleza propia que envuelve la operación de instituciones de investigación científica se encuentra en si misma involucrando comúnmente relaciones socio económicas que permite relacionar su accionar bajo la definición básica de turismo [2]. En este caso, turismo científico.

La contribución económica y social del turismo científico, especialmente en regiones tropicales, ha sido poco estudiada por la academia y por el propio sector turístico; debido a ello hay una gran cantidad de interrogantes sobre el turismo científico, puesto que la categorización o aplicación de los diversos tipos de turismo no ha sido aun uniformemente empleada en los ámbitos territoriales del trópico. Dentro de las instituciones que en América Latina han dedicado esfuerzos para promover el desarrollo y el análisis de la dinámica del turismo científico, destaca el Centro de Turismo Científico de la Patagonia (CTCP) creado en Chile [2].

Los centros de investigación científica de ciencias biológicas representan instituciones de carácter multi o uni disciplinario que facilitan y proveen un aumento tanto en la variedad como en la calidad de información disponible para la población de las comunidades donde se ubican. Los resultados de las investigaciones, dependiendo del enfoque con el que se analicen, pueden llegar a tener importancia a nivel internacional mediante la representación de la realidad y el contexto local, caracterizando a las comunidades o los ambientes alrededor de ellas. Tal disponibilidad de conocimiento puede llegar a convertirse en una herramienta más que puede empezar a utilizarse por un gran número de áreas productivas dentro de las cuales destaca el la actividad turística, como actividad no tradicional del ambiente rural, pero con gran capacidad de interrelación comercial que puede de muchas formas favorecer el desarrollo de las comunidades rurales donde se den las actividades.

La presente investigación aborda los aportes que dos centros de investigación científica en ciencias biológicas ubicados en Brasil y Costa Rica brindan a las comunidades rurales donde se ubican.

\section{Metodología}

Para el desarrollo de la investigación fueron seleccionadas dos instituciones de investigación científica en ciencias biológicas, ubicadas en zonas rurales de Brasil y Costa Rica. Se escogieron el Instituto Uiraçu en Brasil y el Centro Soltis para la Investigación y la Educación en Costa Rica.

La toma de datos se hizo en dos etapas.

La primera etapa consistió en una visita presencial de 23 días en cada uno de los centros de investigación seleccionados, durante el periodo comprendido entre los meses de Noviembre y Diciembre del año 2013. Se utilizó la entrevista a profundidad como medio principal de 
obtención de datos y se utilizaron como informantes claves a los doctores Vitor Osmar Becker y Eugenio González Jiménez, directores respectivamente del Instituto Uiraçu y el Centro Soltis para la Investigación y la Educación en Costa Rica. Los tópicos incluidos en las preguntas de las entrevistas se derivaban de los objetivos específicos del trabajo.

La segunda etapa se llevo a cabo realizando un análisis de los datos obtenidos y haciendo una revisión de literatura obtenida a través de búsquedas de información en formato digital en sitios web y revistas electrónicas especializadas en temas afines a la presente investigación.

\section{Generalidades de los centros de investigación científica de ciencias biológicas}

Los centros de investigación seleccionados comparten algunas coincidencias en cuanto a líneas de trabajo:

- Protección de ecosistemas amenazados o poco conocidos científicamente.

- Favorecimiento a la producción de información nueva, relacionada a ciencias biológicas como hidrología, ecología, historia natural, etología, taxonomía, entre otras.

- Apoyo a las manifestaciones culturales locales.

- Diversifican la economía local.

- Cumplen una labor importante en educación ambiental.

- Benefician la producción y captación de agua potable para comunidades vecinas.

\section{El Instituto Uiraçu (Bahía, Brasil)}

Es un centro de investigación científica privado, localizado en la comunidad de Camacan, en el estado de Bahía en Brasil. Desde su fundación en el año 2001 ha desarrollado la investigación científica en el estado de Bahía, así como la educación ambiental y la conservación de los recursos naturales de uno de los ecosistemas más amenazados del patrimonio natural de Brasil: el Bosque del Atlántico o Mata Atlántica [10], [7].

El Instituto surgió como parte de un proyecto de conservación debido al interés del Dr. Vitor Osmar Becker y su familia por conservar y proteger la reducida extensión del mencionado ecosistema, que actualmente existe a lo largo de la cordillera Serra bonita, sitio donde específicamente se ubican las instalaciones del Instituto (V. Becker, comunicación personal, 18 de noviembre del 2013).

El nombre del instituto hace referencia al águila harpía (Harpia harpyja) que es una de las aves de presa más grandes del mundo y de hecho, la más grande en la región de América Central y Suramérica [1]. Aproximadamente hace medio siglo fue considerada una especie característica de la mata atlántica [8]. Actualmente se le considera una especie poco común de observar en este ecosistema [4], [9] y representa una especie emblemática e inspiradora para los esfuerzos de conservación integral de los ecosistemas a los cuales el instituto dedica sus labores. (V. Becker, comunicación personal, 18 de noviembre del 2013).

El trabajo del Instituto Uiraçu se desarrollo especialmente en tres ámbitos. A saber:

1) Conservación de los ecosistemas

2) Investigación científica

3) Educación ambiental 


\section{Conservación de los ecosistemas}

El personal del instituto Uiraçu es consciente del grave estado de conservación del bioma Mata Atlántica y por esto una gran parte de sus esfuerzos operativos se enfocan principalmente en empezar a garantizar una protección efectiva a las fracciones remanentes de aproximadamente 7500 hectáreas del ecosistema a nivel local.

\section{Investigación científica}

Esta área de acción se enfoca en el gran valor del conocimiento y en la incesante necesidad de actualizarlo y hacerlo disponible a la mayor cantidad de miembros de la población posible. Entre otras razones para crear conciencia y también contar con bases solidas para tomar medidas de protección y seleccionar características elementales para lograr preservar el patrimonio natural de un bioma que hasta la fecha ha sido mínimamente estudiado.

Para la atención de investigadores y visitantes en general, el instituto cuenta con: un Aula o salón de reuniones con capacidad para más de 40 personas, dos laboratorios científicos, acceso a internet, más de 2200 hectáreas de bosque, agua potable, servicio de hospedaje y alimentación, aire acondicionado, cuatro guarda parques y más de $7 \mathrm{~km}$ de senderos,.

Como detalle especial, destaca que el instituto tiene a su disposición la colección de lepidópteros del Dr. Becker, la cual es considerada como la tercer mejor colección privada de ese tipo de insectos a nivel mundial, albergando más de 330000 ejemplares procedentes de más de 25 países.

\section{Educación Ambiental}

Mediante la educación ambiental el instituto pretende aproximarse más a los pobladores y el público en general, estimulando el desarrollo de conciencia y responsabilidad ambiental sobre las características biológicas y la importancia de la Mata Atlántica.

Entre los aportes del Instituto a nivel local es importante mencionar que hasta la fecha en sus instalaciones se han realizado más de quince publicaciones científicas, las cuales han evidenciado la importancia biológica de la Mata Atlántica como bioma en riesgo de desaparecer, develando especies nuevas para la ciencia tanto de plantas como animales, destacando el hecho de que muchas de estas han resultado ser endémicas tanto de Brasil como del ecosistema en mención (V. Becker, comunicación personal, 18 de noviembre del 2013).

Para ejemplificar lo mencionado anteriormente se cita el descubrimiento de una nueva especie de escarabajo, correspondiente a un nuevo género de la familia Cerambycidae, cuyo genero y especie: Uirassu beckeri fueron nombrados en honor al Instituto Uiraçu y a su director [5]. Manteniendo la relación con el tema de especies nuevas también es de suma importancia mencionar el descubrimiento de una nueva especie de anfibio denominada Brachycephalus pulex que hasta la fecha solo ha sido encontrada en la cordillera Serra Bonita [6].

Los programas de educación ambiental desarrollados por el instituto como la celebración de la semana de la ciencia y tecnología, semana de la Mata Atlántica y la Semana del Agua han impactado muy positivamente en la calidad de la educación ambiental que reciben miles de personas anualmente, dentro de las cuales destacan los niños y las personas jóvenes tanto a nivel estatal como local porque a través del instituto se capacitan educadores que posteriormente enseñan a los estudiantes las materias relacionadas con el ambiente de una mejor y más actualizada forma (V. Becker, comunicación personal, 18 de noviembre del 2013). 


\section{Centro Soltis para la Investigación y la Educación (San Juan de Peñas Blancas, Costa Rica)}

Es un centro de investigación científica de carácter privado y multi disciplinario perteneciente a la universidad Texas A\&M. Se ubica en la comunidad de San Juan de San Isidro de Peñas Blancas, en la provincia de Alajuela, Costa Rica (E. González, comunicación personal, 19 de Diciembre de 2013).

El centro inicio formalmente sus operaciones el 18 Junio del 2009 y es el resultado de una donación hecha por el señor William Soltis, egresado en 1955 de la carrera de Ingeniería Mecánica de la Universidad Texas A\&M (E. González, comunicación personal, 19 de Diciembre de 2013).

A través de la donación la familia Soltis logró la Universidad Texas A\&M se estableciera en Costa Rica para desarrollar la investigación científica en el trópico húmedo y ayudar en la conservación de los ecosistemas tropicales de una zona muy poco estudiada de Costa Rica, como lo es la cuenca del rio Peñas Blancas (E. González, comunicación personal, 19 de Diciembre de 2013).

El centro Soltis se ha unido a otras instituciones locales de investigación y protección de los recursos naturales como el Bosque Eterno de los Niños y la zona protectora Arenal-Monteverde, entre otras, manteniendo entre todos la interconexión de una franja boscosa que actúa como un corredor biológico de más de 50000 hectáreas que se extiende hasta las partes altas de la cordillera de Tilarán, alcanzando elevaciones cercanas a los 2000 metros sobre el nivel del mar, y que incluye, entre otras, a la comunidad de Monteverde. Esta zona cuenta con un alto grado de endemismo florístico y faunístico tanto para Costa Rica como para la región centroamericana, así como con la presencia del ecosistema Bosque Nuboso Tropical (E. González, comunicación personal, 19 de Diciembre de 2013).

Con la comunidad vecina el Centro Soltis mantiene una estrecha relación y sus aportes se evidencian mediante las relaciones inter institucionales e inter sectoriales que ha logrado establecer e integrar localmente. Como ejemplos de lo anterior destaca el hecho de que más del noventa y cinco por ciento de los empleados contratados a tiempo completo, viven en la comunidad aledaña y que todos los insumos y materiales requeridos para la operación del centro son obtenidos en las tiendas y supermercados cercanos al centro. También se cuenta con la construcción y la donación de un aula, así como la construcción de nuevas tuberías y la instalación de nuevos tanques de almacenamiento de agua potable para uso comunal mediante un esfuerzo coordinado con la comunidad de San Juan de Peñas Blancas y la organización Ingenieros Sin Fronteras de la Universidad Texas A\&M, el auspicio a la feria científica del Ministerio de Educación Pública en el distrito de Peñas Blancas, el acceso a los estudiantes de la comunidad de realizar giras de campo y visitas educativas a las instalaciones del centro incluso utilizando el servicio de internet de manera gratuita (E. González, comunicación personal, 19 de Diciembre de 2013).

A través del Centro Soltis los líderes comunales y la municipalidad local han encontrado un nuevo espacio en el cual desarrollar sus reuniones y discutir asuntos de planificación y desarrollo social en un ambiente con una adecuada infraestructura y con un apoyo personalizado. Incluso las instalaciones han sido utilizadas como centro de capacitaciones de diversos tipos para la población local como por ejemplo cursos de primeros auxilios y manipulación de alimentos entre otros.

La importancia ecológica del centro y la protección de los ecosistemas a nivel regional se evidencia con las más de tres mil especies de plantas vasculares, cuatrocientas especies de Aves, treinta especies de peces de agua dulce, cincuenta y cinco especies de reptiles y cuarenta y cinco especies de anfibios (E. González, comunicación personal, 19 de Diciembre de 2013). 
El Centro Soltis tiene a su disposición una propiedad de 102 hectáreas, cuyo rango elevacional ronda entre los 425 y los 710 metros sobre el nivel del mar y para el desarrollo de sus actividades de conservación, educación e investigación cuenta con: Un edificio académico de 697 metros cuadrados de construcción, dos laboratorios para investigadores, dos aulas o salones de reuniones con capacidad para cuarenta y veinticinco personas respectivamente, una sala con seis computadoras de uso público, un cuarto de emergencias o primeros auxilios, acceso a internet inalámbrico en todas las instalaciones, servicio de lavandería, un comedor, tres oficinas administrativas, un salón de video conferencia y ocho cabinas totalmente equipadas para hospedar una capacidad máxima de 60 personas (E. González, comunicación personal, 19 de Diciembre de 2013).

\section{Discusión}

Tomando en cuenta la información obtenida durante la visita a cada centro de investigación, se debe de considerar que ambas instituciones han surgido en proximidad a ecosistemas naturales muy poco conocidos y estudiados científicamente, en las cercanías de comunidades con un desarrollo turístico muy incipiente. En el caso costarricense, las comunidades más cercanas al Centro Soltis se encuentran ubicadas a distancias no muy lejanas de importantes centros de desarrollo turístico, como La Fortuna y Monteverde. Esta cercanía podrían motivar proyectos turísticos de carácter científico apoyados por el centro Soltis (hasta el momento la ayuda brindada por el centro Soltis se ha enfocado principalmente en los ámbitos social y ambiental). Las comunidades y los sitios que rodean al Instituto Uiraçu, aún no experimentan un apreciable desarrollo turístico pero si tienen mucho potencial, para el cual el apoyo del Instituto puede ser determinante.

Es importante mencionar que al establecerse, ambas instituciones han enfrentado un proceso de inclusión dentro de los sistemas socioeconómicos de cada comunidad, generando en los pobladores opiniones tanto a favor como en contra. Funcionalmente, al estar operando de manera normal las dos instituciones han llegado a representar un aporte nuevo y no tradicional a las actividades socio económicas locales.

Las comunidades Pau Brasil y Camacan en Brasil y San Juan de Peñas Blancas y la Altura en Costa Rica, situadas en las cercanías de ambos centros de investigación tienen problemas socioeconómicos, como pobreza, desempleo (y, por lo tanto, necesidad de más fuentes de trabajo). Por eso el turismo ofrece oportunidades de creación de empresas y nuevos empleos y, en consecuencia, formas de dinamizar la economía local. Evidentemente, ambos centros de investigación pueden cumplir un papel clave, sobre todo si los proyectos se enfocan en el turismo científico, lo cual le daría a dichos proyectos una ventaja comparativa y diferencial respecto a proyectos desarrollados por otras comunidades rurales. Como ejemplos, se presenta para el Instituto Uiraçu, el caso de la pobreza social que aun se mantiene en muchos sectores del estado de Bahía en Brasil, debido a la crisis de la actividad cacaotera de finales de la década de los 80s y para el Centro Soltis se puede citar en auge turístico que presento la región de la Fortuna de San Carlos a partir de las grandes explosiones del volcán Arenal en 1968.

Desde un inicio, ambas instituciones se han involucrado con las comunidades y por ello se ha dado un proceso de inclusión de sus actividades operativas, dentro del conjunto de actividades socioeconómicas que permiten el mantenimiento de las estructuras productivas de cada comunidad, generando así toda una nueva dinámica operativa en cuanto a la dimensión social, ambiental y económica que todo centro de investigación involucra para operar en su contexto local.

Al hacer referencia a la incursión de los centros de investigación dentro del contexto local, resulta de gran importancia mencionar que los aportes más inmediatos de sus operaciones 
están también relacionados directamente con las características particulares de cada sistema socio económico. Para ejemplificar lo indicado anteriormente se da el caso del Instituto Uiraçu que debido a la destrucción y la pérdida de extensión de la Mata Atlántica, una de las primeras acciones operativas que se llevaron a cabo fue empezar a comprar tierras con el fin de asegurar que los remanentes de bosque no corrieran el riesgo de ser utilizados en actividades distintas a la conservación e investigación, garantizando así mejores condiciones para la resiliencia y la perpetuidad del ecosistema a largo plazo. En el caso del Centro Soltis, dado que los territorios boscosos no estaban bajo un peligro tan grave de desaparecer, una de las primeras acciones operativas puestas en macha fue la de empezar a gestionar una buena relación con la comunidad para que en conjunto se diera una protección más activa, mientras se favorecía cada vez más la producción de información nueva sobre temas diversos como investigaciones en meteorología, hidrología e historia natural relacionada con educación ambiental.

Los resultados obtenidos denotan que el interés y la coordinación institucional que la cada centro de investigación ha presentado para con sus comunidades vecinas, ha sido un factor clave para maximizar los aportes y los beneficios de contar con una institución de ese tipo no tradicional a nivel local.

La existencia de una buena relación entre centros de investigación y las comunidades, favorece mucho el desarrollo de canales de comunicación que al establecer un balance en el flujo de información, favorece las condiciones para que ambas partes se integren, gestando así el establecimiento de una relación de apoyo y beneficio mutuos. En este sentido, la organización comunal adquiere un valor especial para lograr enlazar una mayor y mejor cantidad de actores en las diversas acciones de bien común. En ambas instituciones se permite dentro de sus terrenos la captación de fuentes de agua potable para las comunidades vecinas y una vez más, en ambos casos tales acciones han sido el fruto de una relación coordinada entre las instituciones y las comunidades.

En base a la información obtenida de cada centro, la organización comunal se postula como una de las más básicas características que deben tener las comunidades rurales para poder beneficiarse de múltiples formas de la operación de un centro de investigación en su territorio.

\section{Conclusiones}

Los centros de investigación científica pueden llegar a tener un papel muy importante para complementar las iniciativas existentes o incluso para fomentar la creación de estructuras organizativas nuevas que logren generar una sensibilización multidisciplinaria en la población local y a través de ello contar con mejores condiciones cognitivas para poder planificar y desarrollar un rumbo de acción más estratégicamente definido, hacia el cual los esfuerzos comunales puedan dirigirse para lograr una mejor calidad de vida de los pobladores. Esto siempre y cuando se logre establecer una relación dual balanceada entre comunidad y las instituciones para su mutuo beneficio.

Ante las múltiples necesidades socio económicas que caracterizan las condiciones de muchas zonas rurales, el turismo científico tiene potencial como una acción integradora de actividades productivas mediante formas no tradicionales de generar recursos económicos que garantizan la protección a largo plazo del patrimonio natural y generan condiciones y oportunidades que favorecen el desarrollo sostenible de las comunidades vecinas.

La información obtenida mediante la investigación, muestra como la organización comunal tiene un papel fundamental para que las comunidades puedan maximizar sus beneficios ante la presencia de en sus terrenos de instituciones que desarrollan turismo científico. 
Debido a que la aplicación del turismo científico aun no ha sido ampliamente difundida y estudiada académicamente en los países seleccionados para el estudio, resulta importante investigar más detalladamente las diversas características operativas y la dinámica intersectorial que mediante el efecto multiplicador, el turismo científico puede generar.

\section{Agradecimientos}

De manera especial se agradece a la Vicerrectoría de Investigación y Extensión del Instituto Tecnológico de Costa Rica y al Dr. Vitor Osmar Becker y el Dr. Eugenio González Jiménez. Ambos directores de las instituciones tomadas en cuenta para la investigación.

En cuanto a la elaboración del documento final se gradece a Francisco Rodríguez Barrientos, Cristian Moreira Segura, Daniel Pérez Murillo y Francisco Céspedes Obando. Profesores de la carrera de Gestión del Turismo Rural Sostenible de la Sede Regional del Instituto tecnológico de Costa Rica.

\section{Bibliografía}

[1] Aguiar-Silva, F. H., Sanaiotti, T. M., Jaudoin, O., Srbek-Araujo, A. C., Siqueira, G., \& Banhos, A. (2012). Harpy Eagle sightings, traces and nesting records at the" Reserva Natural Vale", a Brazilian Atlantic Forest remnant in Espirito Santo, Brazil. REVISTA BRASILEIRA DE ORNITOLOGIA, 20(2), 148-155.

[2] Bourlon, F., Mao, P., \& Osorio, M. (2012). El turismo científico en Aysén: un modelo de valorización territorial basado en el patrimonio y ++ actores locales. Explorando las nuevas fronteras del Turismo, Perspectivas de la investigación en turismo, Exploring the new frontiers of tourism-Tourism reseach perspectives, En explorant les nouvelles frontières du tourisme, Perspectives de la recherche en tourisme, 125-143.

[3] Bourlon, F., \& Mao, P. (2011). Las formas del turismo científico en Aysén, Chile. Gest. tur.(Valdivia), (15), 74-98.

[4] Galetti, M.; Martuscelli, P.; Pizo, M. A.; Simão, I. (1997). Records of Harpy and Crested Eagles in the Brazilian Atlantic forest. Bulletin of the British Ornithologists' Club 117: 27-31.

[5] Martins, U. R., \& Galileo, M. H. M. (2010). Cerambycidae (Coleoptera) da Serra Bonita, Camacan, Bahia, Brasil. Papéis Avulsos de Zoologia (São Paulo),50(28), 435-443.

[6] Napoli, M. F., Caramaschi, U. L. I. S. S. E. S., Cruz, C. A. G., \& Dias, I. R. (2011). A new species of flea-toad, genus Brachycephalus Fitzinger (Amphibia: Anura: Brachycephalidae), from the Atlantic rainforest of southern Bahia, Brazil.Zootaxa, 2739, 33-40.

[7] Pardini, R., de Souza, S. M., Braga-Neto, R., \& Metzger, J. P. (2005). The role of forest structure, fragment size and corridors in maintaining small mammal abundance and diversity in an Atlantic forest landscape. Biological conservation, 124(2), 253-266.

[8] Ribon, R., Simon, J. E., \& Theodoro De Mattos, G. (2003). Bird extinctions in Atlantic forest fragments of the Viçosa region, southeastern Brazil.Conservation Biology, 17(6), 1827-1839.

[9] Sánchez-Lalinde, C., Vélez-García, F., Cornélio, A. C., Silveira, L. F. \& Alvarez, M. R. (2011). Records of the Harpy Eagle (Harpia harpyja) in the Serra Bonita reserves complex, Camacan, Bahia, with evidence of breeding. Revista Brasileña de Ornitologia. 19: 436-438.

[10] Viveiros de Castro, E. B., \& Fernandez, F. A. (2004). Determinants of differential extinction vulnerabilities of small mammals in Atlantic forest fragments in Brazil. Biological Conservation, 119(1), 73-80. 